\title{
Programa eco difusiones virtuales y competencias ambientales en estudiantes de educación primaria
}

Teresa Isabel Villarreal Guzmán

tvillarreal@ucvvirtual.edu.pe https://orcid.org/0000-0002-4029-8986

Groberti Alfredo Medina Corcuera

grome@ucvvirtual.edu.pe

https://orcid.org/0000-0003-4035-157X

Universidad César Vallejo

Chimbote- Perú

\section{RESUMEN}

La presente investigación buscó demostrar la incidencia del programa ecodifusiones virtuales en el desarrollo de las competencias ambientales de los estudiantes del V ciclo de la I.E. "Edith Weed Davis", Chimbote, 2021. Fue de tipo aplicada con método cuantitativo y diseño cuasi experimental con una muestra de 31 estudiantes entre grupo experimental y de control. Para la recolección de datos se usó la encuesta como técnica y un cuestionario como instrumento pre test y post test, validado por 2 jueces expertos con una confiabilidad de $\alpha=0,770$ (Alfa de Cronbach). Los resultados alcanzaron niveles desde un $44.4 \%$ en inicio y $50.0 \%$ en proceso con $0 \%$ en nivel destacado en el pre test del grupo experimental hasta un $83.3 \%$ en nivel destacado y $0 \%$ en inicio en el post test. Asimismo, para la prueba de hipótesis se usó la T de Student con un valor de significancia de 0.000 , situado por debajo de 0.05 , aceptando la hipótesis alterna y afirmando que el programa ecodifusiones virtuales incide significativamente en el desarrollo de las competencias ambientales.

Palabras clave: contaminación ambiental; competencias ambientales; eco difusiones virtuales; redes sociales 


\title{
Virtual eco-broadcasting program and environmental competencies in primary school students
}

\begin{abstract}
The present research sought to demonstrate the incidence of the virtual eco-broadcasts program in the development of environmental competencies of the $\mathrm{V}$ cycle students of the E.I. "Edith Weed Davis", Chimbote, 2021. The research was an applied type with a quantitative method and quasi-experimental design with a sample of 31 between experimental and control groups. For data collection, the survey was used as a technique, and a questionnaire as a pre-test and post-test instrument, validated by two expert judges with a reliability of $\alpha=0.770$ (Ctonbach's Alpha). The results reached levels from $44.4 \%$ in the beginning and $50.0 \%$ in the process, with $0 \%$ in the outstanding level in the pretest of the experimental group to $83.3 \%$ in the outstanding level and $0 \%$ in the beginning in the post-test. Likewise, for the hypothesis test, the Student's T distribution was used with a significance value of 0.000 , located below 0.05 , accepting the alternative hypothesis and affirming that the virtual ecodifusiones program has a significant impact on the development of environmental competencies
\end{abstract}

Keywords: environmental pollution; environmental competencies; virtual ecobroadcasts; social networks.

Artículo recibido: 15 noviembre. 2021 Aceptado para publicación: 10 diciembre 2021 Correspondencia: telecho_1730@ hotmail.com.pe Conflictos de Interés: Ninguna que declarar 


\section{INTRODUCCIÓN}

La contaminación ambiental es un problema mundial ocasionado por la falta del desarrollo de competencias ambientales, esto es falta de conocimientos, actitudes y comportamientos ambientales (Severiche-Sierra, Gómez-Bustamante y Jaimes-Morales, 2016). El ser humano es el agente principal que ha contribuido al deterioro de su planeta, no sabe utilizar los recursos y las consecuencias son visibles con el efecto invernadero, cambio climático y desequilibrio ecológico (Camposano, 2019). Estamos inmersos en una realidad totalmente preocupante, a la que algunos autores llaman colapso ambiental o riesgo existencial (Kareiva y Carranza, 2018). Entre los años 2030 y 2050, el cambio climático causará hasta 250000 muertes adicionales por año (World Health Organization [WHO], 2019). Frente a ello, Camposano (2019) señala que es urgente aprovechar un medio y la educación es ese medio que tiene el rol de desarrollar competencias ambientales. Por lo expuesto, hay necesidad de seguir investigando esta problemática tanto en el ámbito educativo como social, es así como surge el problema de investigación: ¿De qué manera el programa ecodifusiones virtuales incide en el desarrollo de las competencias ambientales en los estudiantes del V ciclo de la I.E. Edith Weed Davis, Chimbote, 2021 ?

Asimismo, la propuesta basada en un programa contó con los siguientes criterios para resaltar su importancia: Primero, es conveniente darle atención a una problemática ambiental porque aún persiste; segundo, tiene relevancia social porque contribuirá a cambiar el comportamiento de niños ; tercero, posee implicancias prácticas, pues el desarrollo de estas competencias se hará notorio en cada acción de nuestros niños como protagonistas de cada actividad propuesta y cuarto, la investigación tiene un valor teórico porque con los resultados obtenidos las conclusiones serán un gran aporte teórico para futuras investigaciones interesadas (Fernández - Bedoya, 2020).Además, el presente estudio tuvo como objetivo general: Demostrar la incidencia que presenta el programa ecodifusiones virtuales en el desarrollo de las competencias ambientales de los estudiantes del V ciclo de la I.E. "Edith Weed Davis", Chimbote, 2021. Asimismo, busca cumplir con objetivos específicos como: Comparar los resultados de las dimensiones identificación con su biodiversidad, comprensión de efectos atmosféricos, protección de la biodiversidad, producción y consumo responsable, reflexión del impacto de su huella 
de carbono antes y después de la aplicación del programa y finalmente elaborar una propuesta del programa para el desarrollo de las competencias ambientales (Viñan, Navarrete, Puente, Pino y Caicedo, 2018).

A su vez, contó con una hipótesis alterna descrita así: El programa ecodifusiones virtuales incide significativamente en el desarrollo de las competencias ambientales en los estudiantes del V ciclo de la I.E. Edith Weed Davis, Chimbote, 2021, mientras que la hipótesis nula fue la negación de la alterna (Espinoza, 2018).Existen investigaciones relacionadas al presente estudio, a nivel internacional en una investigación sobre el desarrollo de la conciencia ambiental en niños, Díaz y Fuentes (2018) concluyeron que es necesario continuar con el estudio, análisis y comprensión sobre las formas de lograr reflexión y acción para la conservación de nuestra naturaleza, pues han descubierto que nuestros niños poseen afectos y cogniciones pro ambientales que deben ser aprovechables. A nivel nacional, en Lima, Yangalí, Vásquez, Huaita y Baldeón (2020) realizaron una investigación relacionada al comportamiento ecológico y la cultura ambiental, concluyendo que es posible mejorar el comportamiento ecológico de nuestros educandos y comprometerlos junto a sus familias a seguir realizando acciones a favor del medio ambiente. Este estudio consideró fundamentos teóricos de los proyectos integrados que MINEDU ha propuesto en educación presencial, pero los investigadores lo aplicaron haciendo uso de sus aulas virtuales como único medio de difusión.

En el ámbito regional, Arias (2020) concluyó que la bahía de la ciudad de Coishco se encuentra afectada por líquidos residuales que son provocados por sus industrias pesqueras, y la actividad humana, por lo tanto, es una playa no apta para visitar por recreación y por tener buena carga de microbios en sus orillas. A nivel local, en Chimbote, los autores Quillos, Escalante, Sánchez, Quevedo y De La Cruz (2018) en su estudio sobre estimación energética y caracterización de residuos sólidos domiciliarios, concluyeron que una de las importancias de reciclar y clasificar residuos sólidos como papel, plásticos, vidrio, cartón desde nuestros hogares, sería para una recuperación "energética" a partir de los componentes orgánicos que estos contienen, esto significa obtención de energía que ayudaría a reducir el consumo de combustibles fósiles evitando enfermedades biológicas por su mal uso. En la institución educativa N 88015 "Edith Weed Davis", el problema ambiental se manifestaba cuando sus estudiantes reflejaban en sus conductas la falta de valoración de sus espacios naturales, descuidando primero sus áreas verdes y 
desconociendo las áreas naturales protegidas de su región, también era visible la poca costumbre de reciclar, el consumismo exagerado de productos sin identificar claramente contenedores, la falta del hábito de caminar a pie, entre otras prácticas inadecuadas. Por todo ello fue que se tomó la decisión de investigar proponiendo un programa ambiental como posible solución.

En cuanto a las variables de estudio, la investigación tuvo como variable dependiente a las competencias ambientales, mientras que la independiente fue el programa ecodifusiones virtuales y ambas se sustentaron en teorías que les dieron el debido fundamento. Al respecto, Acero y Ull (como se citaron en Velásquez, Bedoya y Cadavid,2019) definen a las competencias ambientales como las destrezas, habilidades, actitudes y valores en el ser humano para relacionarse de forma sostenible y responsable con su entorno natural y sus elementos, de esta manera enfrentar situaciones problemáticas relacionadas con su ambiente. Sobre las dimensiones, fueron 5 dimensiones basadas en fundamentos teóricos de los Proyectos Educativos Ambientales Integrados (PEAI), una de las propuestas de MINEDU en educación ambiental. Así pues, la primera dimensión fue identificación con su biodiversidad, basada en el proyecto Vida y Verde-Vive y referida al reconocimiento y valoración de espacios naturales, empezando por las áreas naturales protegidas de su ciudad y región.

La segunda dimensión fue la comprensión de efectos atmosféricos que tomó como referencia al proyecto Globe Perú: Conciencia Ambiental desde la escuela y buscó la indagación científica en cuanto a efectos atmosféricos como el cambio climático se refiere e identificación de problemas ambientales que afectan su región y localidad. La tercera dimensión fue protección de la biodiversidad, inspirada en el proyecto Espacio de vida (EsVi) y referida a la creación, recuperación y aprovechamiento de espacios, esto estuvo referido al buen uso de espacios vacíos dentro de sus hogares para dar vida a seres como plantas o animales, protegiendo así su biodiversidad. La cuarta dimensión, consistió en la producción y consumo responsable, sustentada en el proyecto Manejo de Residuos Sólidos en las II.EE (Mares), y referida a la buena gestión de recursos sólidos desde sus hogares, identificando las clases de residuos, el significado de los colores de cada contenedor, conociendo y aplicando las $3 \mathrm{R}$ y por último, la quinta dimensión fue reflexión del impacto de su huella de carbono, basada en el proyecto Mido y Reduzco mi Huella de Carbono y la de mi Cole que buscó que los estudiantes calculen su huella de 
carbono a través de un aplicativo sugerido para reflexionar sobre las consecuencias de sus acciones y estilos de vida con relación a la emisión de gases de efecto invernadero y reducir su huella de carbono. (Ministerio de Educación [MINEDU], 2017).

Asimismo, la investigación se desarrolló bajo un enfoque ambiental, reconocido por el Currículo Nacional de la Educación Básica como uno de los 7 enfoques transversales para el desarrollo del Perfil de egreso de los niños. El enfoque ambiental busca el logro de actitudes y valores visibles en las formas de actuar de cada uno de nuestros estudiantes, dándoles un sentido de vida, y favoreciendo el desarrollo de competencias (Ministerio de Educación [MINEDU], 2020). Con respecto al programa ecodifusiones virtuales, fue un programa ambiental que buscó desarrollar competencias ambientales de nuestros niños y uso de forma educativa a las redes sociales como recursos virtuales para luego difundir cada una de las actividades por tales medios. Aquí los estudiantes generaron sus propios aprendizajes, desarrollando así sus competencias ambientales, que es una construcción consciente que se va logrando poco a poco, de acuerdo a su ciclo y con el apoyo de profesores, instituciones y programas de educación (Ministerio de Educación [MINEDU], 2017). Durante el programa se propuso 14 sesiones con actividades ambientales para contribuir al reconocimiento y valoración de su medio natural, comprensión de problemas ambientales y reflexión para el cuidado de su medio ambiente, usando diversas estrategias. Además, estuvo fundamentado por la teoría de Usos y Gratificaciones (UyG), donde Dong y Day (como se citaron en García-Ruíz, Tirado y Hernando, 2018) manifestaron que los individuos realizan una selección de manera activa de los medios de comunicación en función de sus intereses y necesidades con la finalidad de satisfacer sus deseos y obtener una recompensa.

\section{ESTRATEGIAS METODOLÓGICAS O MATERIALES Y MÉTODOS}

El estudio realizado fue una investigación de tipo aplicada, con método cuantitativo y diseño cuasi experimental, donde tanto grupo control como experimental recibieron el pre y post test, pero solamente el grupo experimental recibió el tratamiento (variable independiente) y se esperó el cambio o la variación de éste en la variable dependiente. El diagrama del diseño en mención fue el siguiente: 


\section{Donde:}

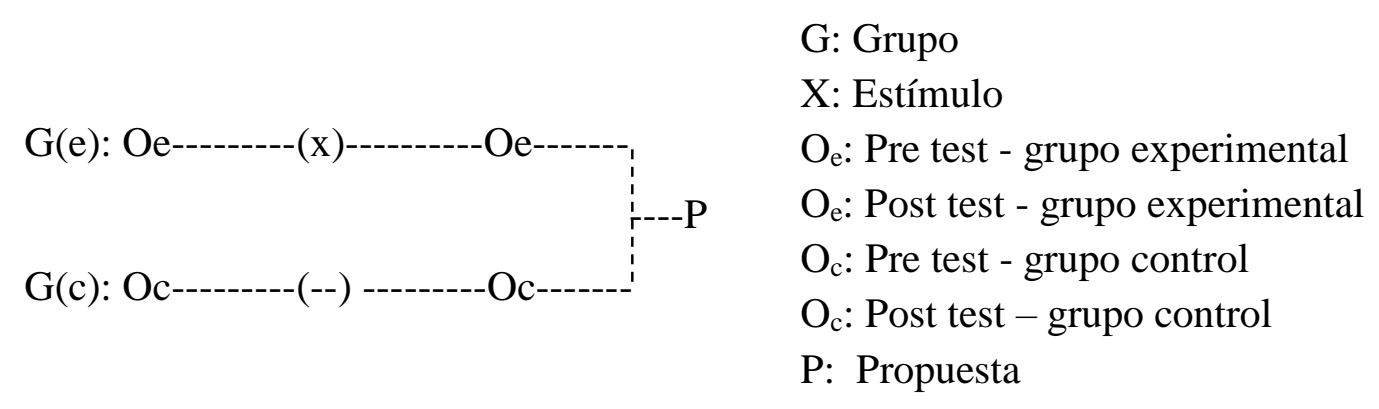

El estudio tuvo una población de 305 estudiantes de la I.E. Edith Weed Davis y su muestra estuvo conformada por 31 educandos del V ciclo de Educación Primaria donde 18 niños integraron el grupo experimental y 13 estudiantes conformaron el grupo control. Para evaluar el nivel de competencias ambientales de la muestra, se aplicó la técnica de la encuesta con un cuestionario como instrumento pre test y post test, el mismo que luego de ser creado pasó a ser validado por juicios de expertos de 2 especialistas y luego evaluado con la base de una prueba piloto con la participación de 10 estudiantes para su confiabilidad con el Alfa de Cronbach, resultando confiable en un $\alpha=0.770$, siendo aceptable.

Sobre el cuestionario, contenía 20 ítems con preguntas objetivas y opción de respuesta desde la alternativa $\mathrm{A}$ hasta la $\mathrm{D}$, ítems que a su vez pertenecieron a 5 indicadores propios de las cinco dimensiones de la variable dependiente, donde 4 ítems fueron para la dimensión identificación con su biodiversidad, 4 para la dimensión comprensión de efectos atmosféricos, 4 más para protección de la biodiversidad , 4 ítems para la dimensión producción y consumo responsable, y 4 últimos ítems más para reflexión del impacto de su huella de carbono. En cuanto a los niveles de las competencias ambientales y sus puntajes, fueron 4 niveles: N. inicio (0-1), N. proceso (2), N. logro esperado (3) y N. destacado (4).

Sobre el procesamiento estadístico, se hizo uso de la estadística descriptiva con una base de datos que facilitó el procesamiento estadístico inferencial con la prueba de normalidad de Shapiro-Wilk, considerado para muestras menores a 50, por otro lado al realizar un análisis del promedio de la significancia alcanzada en el pre y pos test de las competencias ambientales se tuvo el siguiente calculo $(0.136+0.001) / 2=0.0685$, situado por encima del 0.05 , con lo que dio a entender que la muestra presentaba una distribución paramétrica y el método más adecuado para conocer el efecto de la variable independiente sobre la dependiente era la T de Student. 


\section{RESULTADOS Y DISCUSIÓN}

\subsection{Resultados}

\section{Análisis descriptivo}

\section{Tabla 1}

Frecuencia de la incidencia que presenta el programa ecodifusiones virtuales en el desarrollo de competencias ambientales en los estudiantes.

\begin{tabular}{|c|c|c|c|c|c|}
\hline \multirow{7}{*}{ 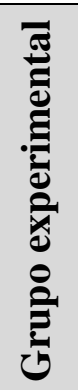 } & \multirow{2}{*}{ Niveles } & \multicolumn{2}{|c|}{ Pre Test } & \multicolumn{2}{|c|}{ Post Test } \\
\hline & & fi & $\%$ & fi & $\%$ \\
\hline & Inicio & 8 & $44.4 \%$ & 0 & $0.0 \%$ \\
\hline & Proceso & 9 & $50.0 \%$ & 1 & $5.6 \%$ \\
\hline & Logro Esperado & 1 & $5.6 \%$ & 2 & $11.1 \%$ \\
\hline & Logro Destacado & 0 & $0.0 \%$ & 15 & $83.3 \%$ \\
\hline & Total & 18 & $100.0 \%$ & 18 & $100.0 \%$ \\
\hline \multirow{7}{*}{ 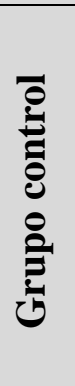 } & \multirow{2}{*}{ Niveles } & \multicolumn{2}{|c|}{ Pre Test } & \multicolumn{2}{|c|}{ Post Test } \\
\hline & & fi & $\%$ & fi & $\%$ \\
\hline & Inicio & 1 & $7.7 \%$ & 1 & $7.7 \%$ \\
\hline & Proceso & 10 & $76.9 \%$ & 8 & $61.5 \%$ \\
\hline & Logro Esperado & 2 & $15.4 \%$ & 4 & $30.8 \%$ \\
\hline & Logro Destacado & 0 & $0.0 \%$ & 0 & $0.0 \%$ \\
\hline & Total & 13 & $100.0 \%$ & 13 & $100.0 \%$ \\
\hline
\end{tabular}

Fuente: Base de datos del pre y post test del grupo experimental y control

\section{Interpretación:}

En la tabla 1, el grupo experimental registró en nivel de inicio del pre test a $8(44.4 \%)$ estudiantes, pero ya en el post test se registró (0.0\%). Por otro lado, el grupo control tuvo un resultado sorprendente de tan solo 1 estudiante $(7.7 \%)$ en el pre test y se mantuvo con ese mismo valor en el post test. En el nivel proceso, tanto grupo experimental como de control, durante el pre test, mantuvieron similar resultado; 9 (50.0\%) y $10(76.9 \%)$ estudiantes respectivamente, luego en el post test el resultado fue favorable con tan solo $1(5.6 \%)$ estudiante para el experimental, pero 8 (61.5\%) para el de control. Además, en cuanto a logro esperado, el experimental en pre test tuvo a $1(5.6 \%)$ educando, y en pos test a $2(11.2 \%)$, entretanto el grupo control obtuvo en pre test a $2(15.4 \%)$ y post test a 4 (30.8\%). Finalmente, en logro destacado, el grupo experimental no presenta datos en el pre test, pero en el post test sí tiene un resultado muy favorable de 15 (83.3\%), quedando el grupo control con $0.0 \%$ tanto en pre como post test durante este nivel. 


\section{Tabla 2}

Frecuencia de los resultados de la dimensión identificación con su biodiversidad antes y después de la aplicación del programa ecodifusiones virtuales en los estudiantes.

\begin{tabular}{|c|c|c|c|c|c|}
\hline \multirow{7}{*}{ 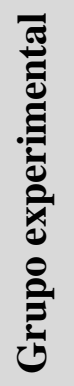 } & \multirow{2}{*}{ Niveles } & \multicolumn{2}{|c|}{ Pre Test } & \multicolumn{2}{|c|}{ Post Test } \\
\hline & & fi & $\%$ & fi & $\%$ \\
\hline & Inicio & 15 & $83.3 \%$ & 1 & $5.6 \%$ \\
\hline & Proceso & 1 & $5.6 \%$ & 1 & $5.6 \%$ \\
\hline & Logro Esperado & 2 & $11.1 \%$ & 5 & $27.8 \%$ \\
\hline & Logro Destacado & 0 & $0.0 \%$ & 11 & $61.1 \%$ \\
\hline & Total & 18 & $100.0 \%$ & 18 & $100.0 \%$ \\
\hline \multirow{7}{*}{ 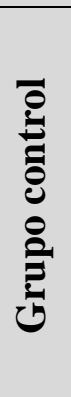 } & Niveles & \multicolumn{2}{|c|}{ Pre Test } & \multicolumn{2}{|c|}{ Post Test } \\
\hline & N1veles & fi & $\%$ & fi & $\%$ \\
\hline & Inicio & 10 & $76.9 \%$ & 9 & $69.2 \%$ \\
\hline & Proceso & 3 & $23.1 \%$ & 4 & $30.8 \%$ \\
\hline & Logro Esperado & 0 & $0.0 \%$ & 0 & $0.0 \%$ \\
\hline & Logro Destacado & 0 & $0.0 \%$ & 0 & $0.0 \%$ \\
\hline & Total & 13 & $100.0 \%$ & 13 & $100.0 \%$ \\
\hline
\end{tabular}

Fuente: Base de datos del pre y post test del grupo experimental y control

\section{Interpretación:}

La tabla 2 muestra que en la dimensión identificación con su biodiversidad, tanto el grupo experimental como el de control señalan un puntaje parecido de $15(83.3 \%)$ y $10(76.9 \%)$ niños en el nivel inicio del pre test, ya en el post test, el valor de $1(5.6 \%)$ estudiante para el experimental resulta muy diferenciado de $9(69.2 \%)$ en el grupo control. Detallando el nivel proceso, los datos obtenidos fueron más bajos, pues el grupo experimental indica un mismo resultado en el pre y post test de $1(5.6 \%)$ y el grupo control arroja primero a 3 (23.1\%), y después a $4(30.8 \%)$ niños. En relación al nivel logro esperado, el grupo experimental registró en el pre test a $2(11.1 \%)$, mientras que en el pos test a $5(27.8 \%)$, frente a un grupo control que no evidencia registro. En el nivel logro destacado, el experimental, aunque no presenta puntaje en el pre test; en el post test se visualiza un valor positivo de $11(61.1 \%)$, esto difiere mucho del grupo control donde no se presenta registro ni en pre ni post test. 


\section{Tabla 3}

Frecuencia de los resultados de la dimensión comprensión de efectos atmosféricos antes y después de la aplicación del programa ecodifusiones virtuales en los estudiantes.

\begin{tabular}{|c|c|c|c|c|c|}
\hline \multirow{7}{*}{ 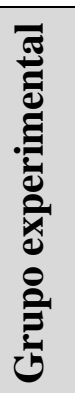 } & \multirow{2}{*}{ Niveles } & \multicolumn{2}{|c|}{ Pre Test } & \multicolumn{2}{|c|}{ Post Test } \\
\hline & & fi & $\%$ & fi & $\%$ \\
\hline & Inicio & 11 & $61.1 \%$ & 2 & $11.1 \%$ \\
\hline & Proceso & 5 & $27.8 \%$ & 0 & $0.0 \%$ \\
\hline & Logro Esperado & 2 & $11.1 \%$ & 3 & $16.7 \%$ \\
\hline & Logro Destacado & 0 & $0.0 \%$ & 13 & $72.2 \%$ \\
\hline & Total & 18 & $100.0 \%$ & 18 & $100.0 \%$ \\
\hline \multirow{7}{*}{ 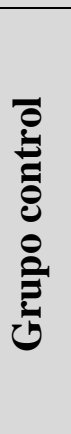 } & \multirow{2}{*}{ Niveles } & \multicolumn{2}{|c|}{ Pre Test } & \multicolumn{2}{|c|}{ Post Test } \\
\hline & & $\mathbf{f i}$ & $\%$ & fi & $\%$ \\
\hline & Inicio & 7 & $53.8 \%$ & 7 & $53.8 \%$ \\
\hline & Proceso & 5 & $38.5 \%$ & 5 & $38.5 \%$ \\
\hline & Logro Esperado & 1 & $7.7 \%$ & 1 & $7.7 \%$ \\
\hline & Logro Destacado & 0 & $0.0 \%$ & 0 & $0.0 \%$ \\
\hline & Total & 13 & $100.0 \%$ & 13 & $100.0 \%$ \\
\hline
\end{tabular}

Fuente: Base de datos del pre y post test del grupo experimental y control

\section{Interpretación:}

La tabla 3 detalla que en la dimensión comprensión de efectos atmosféricos, 11 (61.1\%) estudiantes del grupo experimental están en inicio del pre test, mientras que en el pos test favorablemente disminuye a 2 (11. 1\%). Por otro lado, una igualdad de 7 (53.8\%) niños del grupo control fue dada tanto en el pre test como en el post test de este nivel. En el nivel proceso se ubicaron 5 (27.8\%) niños del experimental en el pre test, pero en el pos test este nivel no es alcanzado por ningún estudiante, en cuanto al control, hubo en el pre test a $5(38.5 \%)$, mientras que en el pos test $0(0.0 \%)$. Con respecto al nivel logro esperado, el experimental primero alcanzó a 2 (11.1\%), mientras que en el pos test se tuvo a $3(16.7 \%)$, frente a un grupo control que tuvo un nivel igualado tanto en pre test y post test de $1(7.7 \%)$ educando .Por último, en el nivel logro destacado del grupo experimental no se presentan datos en el pre test, mientras que en el post test se tuvo como resultado positivo a $13(72.2 \%)$, en cambio al mencionar este nivel en el grupo control no se presenta registro en el pre test y tampoco en el pos test. 


\section{Tabla 4.}

Frecuencia de los resultados de la dimensión protección de la biodiversidad antes y después de la aplicación del programa ecodifusiones virtuales en los estudiantes.

\begin{tabular}{|c|c|c|c|c|c|}
\hline \multirow{7}{*}{ 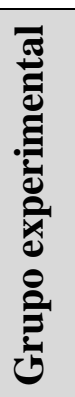 } & \multirow{2}{*}{ Niveles } & \multicolumn{2}{|c|}{ Pre Test } & \multicolumn{2}{|c|}{ Post Test } \\
\hline & & fi & $\%$ & fi & $\%$ \\
\hline & Inicio & 8 & $44.4 \%$ & 1 & $5.6 \%$ \\
\hline & Proceso & 7 & $38.9 \%$ & 1 & $5.6 \%$ \\
\hline & Logro Esperado & 3 & $16.7 \%$ & 2 & $11.1 \%$ \\
\hline & Logro Destacado & 0 & $0.0 \%$ & 14 & $77.8 \%$ \\
\hline & Total & 18 & $100.0 \%$ & 18 & $100.0 \%$ \\
\hline \multirow{7}{*}{ 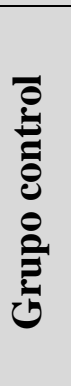 } & \multirow{2}{*}{ Niveles } & \multicolumn{2}{|c|}{ Pre Test } & \multicolumn{2}{|c|}{ Post Test } \\
\hline & & fi & $\%$ & fi & $\%$ \\
\hline & Inicio & 5 & $38.5 \%$ & 5 & $38.5 \%$ \\
\hline & Proceso & 7 & $53.8 \%$ & 6 & $46.2 \%$ \\
\hline & Logro Esperado & 0 & $0.0 \%$ & 1 & $7.7 \%$ \\
\hline & Logro Destacado & 1 & $7.7 \%$ & 1 & $7.7 \%$ \\
\hline & Total & 13 & $100.0 \%$ & 13 & $100.0 \%$ \\
\hline
\end{tabular}

Fuente: Base de datos del pre y post test del grupo experimental y control

\section{Interpretación:}

La tabla 4 explica los resultados obtenidos en la tercera dimensión protección de la biodiversidad demostrando que $8(44.4 \%)$ estudiantes del grupo experimental estaban en el nivel inicio del pre test, pero que en el pos test positivamente hubo 1 (5.6\%), y en comparación con el control se observó a 5 (38.5\%) niños tanto en el pre test como el post test. Observando el nivel proceso se encontró a 7 (38.9\%) estudiantes en el experimental durante el pre test y en el post test solo a 1 (5.6\%). Además, el grupo control en el pre test presentó a 7 (53.8\%) niños y en el post test se tuvo a 6 (46.2\%). Con respecto al nivel logro esperado, el grupo experimental alcanzó en el pre test un puntaje de $3(16.7 \%)$ educandos, mientras que en el post test solo 2 (11.1\%) niños lo alcanzaron, en referencia al grupo control, en el pre test ningún estudiante lo alcanzó, y que en el post test solo 1 (7.7\%). Para finalizar, el experimental en el nivel logro destacado no presentó datos en el pre test, pero en el post test este nivel sí fue alcanzado por 14 (77.7\%), mientras que en el grupo control durante el pre y post test solo 1 (7.7\%) estudiante quedó registrado. 


\section{Tabla 5.}

Frecuencia de los resultados de la dimensión producción y consumo responsable antes y después de la aplicación del programa ecodifusiones virtuales en los estudiantes.

\begin{tabular}{|c|c|c|c|c|c|}
\hline \multirow{7}{*}{ 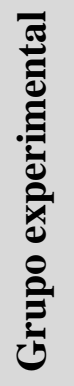 } & \multirow{2}{*}{ Niveles } & \multicolumn{2}{|c|}{ Pre Test } & \multicolumn{2}{|c|}{ Post Test } \\
\hline & & fi & $\%$ & fi & $\%$ \\
\hline & Inicio & 9 & $50.0 \%$ & 0 & $0.0 \%$ \\
\hline & Proceso & 8 & $44.4 \%$ & 3 & $16.7 \%$ \\
\hline & Logro Esperado & 1 & $5.6 \%$ & 3 & $16.7 \%$ \\
\hline & Logro Destacado & 0 & $0.0 \%$ & 12 & $66.7 \%$ \\
\hline & Total & 18 & $100.0 \%$ & 18 & $100.0 \%$ \\
\hline \multirow{7}{*}{ 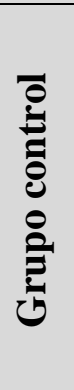 } & \multirow{2}{*}{ Niveles } & \multicolumn{2}{|c|}{ Pre Test } & \multicolumn{2}{|c|}{ Post Test } \\
\hline & & $\mathbf{f i}$ & $\%$ & fi & $\%$ \\
\hline & Inicio & 0 & $0.0 \%$ & 1 & $7.7 \%$ \\
\hline & Proceso & 5 & $38.5 \%$ & 4 & $30.8 \%$ \\
\hline & Logro Esperado & 6 & $46.2 \%$ & 5 & $38.5 \%$ \\
\hline & Logro Destacado & 2 & $15.4 \%$ & 3 & $23.1 \%$ \\
\hline & Total & 13 & $100.0 \%$ & 13 & $100.0 \%$ \\
\hline
\end{tabular}

Fuente: Base de datos del pre y post test del grupo experimental y control

\section{Interpretación:}

La tabla 5 indica valores alcanzados de los estudiantes en la dimensión producción y consumo responsable y detalla que 9 (50.0\%) niños del grupo experimental se encontraron en inicio del pre test, pero en el post test ninguno lo alcanzó, luego hablando del grupo control, durante el pre test y post test solamente1 (7.7\%) es el estudiante que se encontró. En el nivel de proceso, el experimental tuvo como valores en el pre test a 8 (44.4\%) estudiantes, mientras que en el post test a $3(16.7 \%)$ y control tuvo en el pre test a $5(38.5 \%)$, mientras que en el pos test a 4 (30.8\%). Con relación al nivel logro esperado se tiene en el pre test del grupo experimental a $1(5.6 \%)$ y en el post test a $3(16.7 \%)$ respectivamente. Luego en el grupo control se tuvo en el pre test a $6(46.2 \%)$, mientras que en el post test a $5(38.5 \%)$.En cuanto a logro destacado no se presentan datos en el pre test del experimental, mientras que en el post test se tuvo a 12 (66.7\%), y en el control se tuvo en el pre test a $2(15.4 \%)$, mientras que en el post test a $3(23.1 \%)$. 


\section{Tabla 6.}

Frecuencia de los resultados de la dimensión reflexión del impacto de su huella de carbono antes y después de la aplicación del programa ecodifusiones virtuales en los estudiantes.

\begin{tabular}{|c|c|c|c|c|c|}
\hline \multirow{7}{*}{ 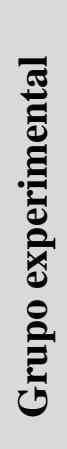 } & \multirow{2}{*}{ Niveles } & \multicolumn{2}{|c|}{ Pre Test } & \multicolumn{2}{|c|}{ Post Test } \\
\hline & & fi & $\%$ & $\mathbf{f i}$ & $\%$ \\
\hline & Inicio & 14 & $77.8 \%$ & 0 & $0.0 \%$ \\
\hline & Proceso & 1 & $5.6 \%$ & 3 & $16.7 \%$ \\
\hline & Logro Esperado & 3 & $16.7 \%$ & 8 & $44.4 \%$ \\
\hline & Logro Destacado & 0 & $0.0 \%$ & 7 & $38.9 \%$ \\
\hline & Total & 18 & $100.0 \%$ & 18 & $100.0 \%$ \\
\hline \multirow{7}{*}{ 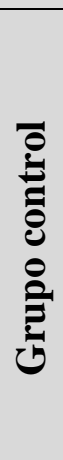 } & \multirow{2}{*}{ Niveles } & \multicolumn{2}{|c|}{ Pre Test } & \multicolumn{2}{|c|}{ Post Test } \\
\hline & & fi & $\%$ & fi & $\%$ \\
\hline & Inicio & 9 & $69.2 \%$ & 7 & $53.8 \%$ \\
\hline & Proceso & 1 & $7.7 \%$ & 2 & $15.4 \%$ \\
\hline & Logro Esperado & 3 & $23.1 \%$ & 4 & $30.8 \%$ \\
\hline & Logro Destacado & 0 & $0.0 \%$ & 0 & $0.0 \%$ \\
\hline & Total & 13 & $100.0 \%$ & 13 & $100.0 \%$ \\
\hline
\end{tabular}

Base de datos del pre y post test del grupo experimental y control

\section{Interpretación:}

La tabla 6 describe datos obtenidos en la dimensión reflexión del impacto de su huella de carbono, donde $14(77.8 \%)$ educandos del grupo experimental en el pre test estaban en inicio, no habiendo ningún estudiante en la evaluación del post test. En cambio, en el grupo control se aprecian resultados similares de $9(69 \%)$ y 7 (53.8\%) entre pre y post test. En el nivel proceso, tanto grupo experimental como de control indican 1 (5.6\%) en pre test, además de similitud de datos en el post test de $3(16.7 \%)$ y 2 (11. 1\%).También en nivel logro esperado, tanto en grupo control como experimental hay una igualdad en pre test de $3(16.7 \%)$ niños para cada uno y respecto al post test se aprecia una superación de $8(44.4 \%)$ educandos del experimental frente a $4(30.8 \%)$ del grupo control. Finalmente, en el nivel logro destacado no se presentan datos en el pre test, mientras que en el post test se tiene a 7 (38.9\%), y al mencionar resultados obtenidos del grupo control, no se registraron datos en el pre test ni en el post test. 


\section{Análisis inferencial}

\section{Tabla 7.}

\section{Prueba de hipótesis}

Hi: El programa ecodifusiones virtuales incide significativamente en el desarrollo de las competencias ambientales en los estudiantes del V ciclo de la I.E Edith Weed Davis, Chimbote, 2021.

Ho: El programa ecodifusiones virtuales no incide significativamente en el desarrollo de las competencias ambientales en los estudiantes del V ciclo de la I.E Edith Weed Davis, Chimbote, 2021.

Nivel de significancia: $\alpha=0.05$

Estadístico de prueba: T de Student

\begin{tabular}{cccccc}
\hline \multirow{2}{*}{ Variable } & \multicolumn{3}{c}{ Prueba $\mathbf{T}-$ Student } & $\begin{array}{c}\text { Nivel de } \\
\text { significancia }\end{array}$ & Decisión \\
& $\begin{array}{c}\text { Valor } \\
\text { observado }\end{array}$ & $\begin{array}{c}\text { Valor } \\
\text { tabular }\end{array}$ & $\begin{array}{c}\text { Probabilidad } \\
\text { significancia }\end{array}$ & & $\begin{array}{c}\mathbf{t}_{\mathbf{0}}>\mathbf{t}_{\mathbf{c}} \\
\mathbf{p}<\alpha\end{array}$ \\
\hline
\end{tabular}

$\begin{gathered}\text { Competencias } \\ \text { ambientales }\end{gathered} \mathrm{t}_{\mathrm{o}}=11,313 \quad \mathrm{t}_{\mathrm{c}}=1,739 \quad \mathrm{p}=0,0000 \quad \alpha=0,05 \quad$ Se rechaza $H_{0}$

Fuente: Base de datos.

\section{Interpretación:}

Para realizar el análisis de la prueba de hipótesis se tiene la prueba T de Student, donde se ha logrado obtener un valor T observado de 11,313 considerado como superior al valor T tabular 1,739 (17 grados de libertad), además al analizar el valor de la significancia obtenida se tiene un valor de 0.000 , situado por debajo del 0.05 con lo cual se procede al rechazo de la hipótesis nula y se comprueba la hipótesis alterna afirmando que el programa ecodifusiones virtuales incide significativamente en el desarrollo de las competencias ambientales en los estudiantes del V ciclo de la I.E Edith Weed Davis, Chimbote, 2021.

\subsection{DISCUSIÓN}

Sabiendo que el presente estudio buscó demostrar la incidencia del programa ecodifusiones virtuales para desarrollar competencias ambientales en niños, los resultados detallan que la mayoría de estudiantes de ambos grupos, en el pre test, en cuanto al desarrollo de sus competencias ambientales, estaban con alto porcentaje tanto en nivel inicio como proceso, por lo que requerían acompañamiento durante un tiempo razonable 
para lograrlo. Por lo expuesto, se puede explicar que los altos valores en nivel proceso e inicio, en el pre test responden a la afirmación que hace MINEDU sobre el desarrollo de las competencias cuando afirma que es una construcción consciente que se va logrando poco a poco (proceso) con el apoyo de profesores, instituciones y programas de educación (Ministerio de Educación [MINEDU], 2017). Asimismo, estos resultados nos permiten afirmar que los niños ya poseían ciertos conocimientos previos en algunos contenidos de las dimensiones propuestas para las competencias ambientales a desarrollar, es decir poseían aquello que Ausubel llamó lo que los niños saben y que el docente debe conocer para facilitar el camino a adquirir un aprendizaje significativo (Fong, Pitre \& Chiquillo Rodelo, 2018).

Luego, los resultados en el post test del grupo experimental alcanzaron altos porcentajes en nivel logro destacado y logro esperado, demostrando que es posible desarrollar competencias ambientales en nuestros niños integrando los proyectos que propone MINEDU en lo que respecta al enfoque ambiental y muestra de la efectividad lo pueden dar los autores Yangalí, Vásquez, Huaita y Baldeón (2020) quienes, en su investigación con variables como el comportamiento ecológico y la cultura ambiental, obtuvieron resultados favorables usando también medios virtuales que en ese caso fueron solamente plataformas para desarrollar las sesiones, difiriendo del presente estudio en donde además se difundieron todas las actividades realizadas por los niños a través de sus redes sociales como Facebook y wasap. Con respecto a los puntajes de la dimensión identificación con su biodiversidad, se tuvo que la mayor parte de estudiantes de ambos grupos se encontraban en nivel inicio y proceso por lo que se infiere que no reconocían como espacios naturales a su bahía, a los humedales, etc. a nivel local menos a nivel regional como sus áreas naturales protegidas, por lo tanto, no se identificaban con su medio natural que los rodea. Al respecto, Rojas (2017) en su ensayo sobre Chimbote afirmó que es una ciudad que crece siendo indiferente con su bahía. Tras la aplicación del programa, solamente los valores obtenidos en el post test del grupo experimental se elevaron a nivel logro destacado y logro esperado.

En la dimensión comprensión de efectos atmosféricos, durante el pre test los puntajes continuaron siendo elevados en el nivel de inicio y proceso para ambos grupos. Ya en el post test los puntajes en el grupo experimental aumentaron en nivel logro destacado y logro esperado. Por lo detallado, se afirma que la mayoría de los estudiantes del grupo 
experimental lograron identificar efectos atmosféricos que afectan su localidad y comprenderlos a través de la realización de experimentos por medio de la investigación, tal como el Ministerio de Educación (MINEDU, 2020) planteó en su proyecto GLOBE Perú: Conciencia ambiental desde la escuela. Asimismo, en la dimensión denominada protección de la biodiversidad, se apreció una vez más que la mayoría de estudiantes de ambos grupos, en el pre test, se encontraban en el nivel inicio y proceso, a su vez cabe resaltar aquí que hubo dos resultados que, aunque menores fueron sorprendentes en el nivel logro esperado del grupo experimental y uno en logro destacado del grupo control. Por lo que se afirma que nuestros niños desde ya poseen afectos y cogniciones pro ambientales que deben ser aprovechables (Díaz y Fuentes, 2018).

Con referencia a la dimensión producción y consumo responsable, en el pre test del grupo experimental los resultados fueron elevados en los niveles de inicio, proceso alcanzando 1 en nivel logro esperado, y sorprendentemente en el grupo control los estudiantes alcanzaron nivel logro esperado, nivel proceso hasta logro destacado. Por lo que se confirma que en esta dimensión nuestros niños tenían mayor conocimiento sobre temas como consumo responsable, residuos sólidos y reciclaje, es que el sistema educativo peruano ha mostrado esfuerzos y ha reforzado estos temas en educación ambiental, aunque no siempre la educación ambiental es llevada a cabo con efectividad en las aulas (Villanueva, Medina y Sánchez, 2020), esta última afirmación sería el motivo del por qué los resultados no fueron totalmente efectivos en el pre test, pero luego de aplicar el programa, los resultados del grupo experimental aumentaron a nivel logro destacado y nivel logro.

Sobre la dimensión reflexión del impacto de su huella de carbono, ambos grupos, en su mayoría se encontraron en nivel inicio, es decir desconocían sobre la medida ecológica huella de carbono que les permitiría reflexionar sobre sus acciones, pero después de la aplicación del programa, los resultados cambiaron para el grupo experimental alcanzando nivel logro destacado, logro esperado, aunque también nivel proceso, por lo que se interpreta que la mayoría de niños pudieron acceder al aplicativo recomendado, pero algunos no pudieron medir su huella de carbono para luego participar de actividades propuestas y fue importante porque como dice Soto (como se citó en Torres, Carbo y López, 2017) les permite asumir un cambio de actitud ambientalmente responsable. Finalmente, los resultados obtenidos en la prueba de hipótesis, permiten a través de la T 
de Student obtener un valor de significancia de 0,000; valor que está situado por debajo del 0.05 , rechazando la hipótesis nula y aceptando la alterna que afirmando que el programa ecodifusiones virtuales incide significantemente en el desarrollo de las competencias ambientales de los estudiantes del V ciclo de la I.E. Edith Weed Davis, Chimbote, 2021. Díaz y Fuentes (2018) se refieren a la efectividad cuando afirman que se necesita continuar con la aplicación de proyectos ambientales. pero que sean innovadores en sus metodologías didácticas.

\section{CONCLUSIONES}

-Se concluyó que el programa ecodifusiones virtuales incide significativamente en el desarrollo de las competencias ambientales, así se evidencia en los resultados de la tabla 11 donde el valor de la significancia de la variable dependiente obtenida fue de 0.000 . Aceptándose la hipótesis alterna.

-Se concluyó que el programa ecodifusiones virtuales fue efectivo en la dimensión identificación con su biodiversidad del grupo experimental, pues los resultados en el post test aumentaron a $61.1 \%$ (11) de niños en nivel logro destacado, seguido de $27.8 \%$ (5) en logro esperado y tan solo $5.6 \%$ (1) tanto en proceso como en inicio.

-Se concluyó que el programa Ecodifusiones Virtuales fue efectivo en la dimensión comprensión de efectos atmosféricos en el grupo experimental, ya que los valores en el post test fueron de 72,2\% (13) de niños en el nivel logro destacado,16.7\% (3) en logro esperado y tan solo $11.1 \%$ (2) en nivel inicio.

-Se concluyó que el programa Ecodifusiones Virtuales fue efectivo en la dimensión protección de la biodiversidad del grupo experimental, pues el post test mostró un elevado $77.8 \%$ (14) de niños en el nivel logro destacado, seguido de $11.1 \%$ (2) en nivel logro esperado y $5.6 \%$ (1) tanto para nivel proceso como nivel inicio.

-Se concluyó que el programa Ecodifusiones Virtuales fue efectivo en la dimensión producción y consumo responsable del grupo experimental, ya que en el post test se alcanzó el $66.7 \%$ (12) de educandos en nivel logro destacado y un $16.7 \%$ (3) de niños tanto para nivel logro esperado como el nivel proceso.

-Se concluyó que el programa ecodifusiones virtuales fue efectivo en la dimensión reflexión del impacto de su huella de carbono del grupo experimental, pues en el post test los puntajes se elevaron a $38.9 \%$ (7) de niños en nivel logro destacado seguido de un $44.4 \%$ (8) en logro esperado y un $16,7 \%$ (3) en nivel de proceso. 
-Se elaboró una propuesta de programa basado en los fundamentos teóricos de los Proyectos Educativos Ambientales Integrados (PEAI) para desarrollar competencias ambientales y que puedan ser contextualizados y considerados por directivos y docentes en sus planificaciones curriculares como estrategias innovadoras.

\section{5.- LISTA DE REFERENCIAS}

Arias, J. (2020). Influencia del vertimiento de los efluentes de la industria pesquera en el agua de mar de la bahía de Coishco, Ancash, en los años 2015 y 2016 (tesis de maestría). Universidad Nacional del Santa, Chimbote, Perú.

Camposano, P. (2019). Actitudes de conservación del ambiente para desarrollar la conciencia ambiental en estudiantes de una Institución Educativa de Huancayo, 2018 (tesis de maestria). Universidad Nacional de Huancavelica, Huancavelica, Perú.

Díaz, J. y Fuentes,F. (2018). Desarrollo de la conciencia ambiental en niños de sexto grado de educación primaria. Significados y percepciones. Revista de Investigación Educativa,(26).Recuperado de http://www.scielo.org.mx/scielo.php?script=sci_arttext\&pid=S187053082018000100136

Espinoza, E. (2018). La hipótesis en la investigación. Mendive: Revista de Educación, 16 (1),122-139. Recuperado de https://mendive.upr.edu.cu/index.php/MendiveUPR/article/view/1197/pdf

Fernández - Bedoya, V. (2020). Tipos de justificación en la investigación científica. Espíritu Emprendedor TEST 2020,4 (3), 65-76.doi: https://doi.org/10.33970/eetes.v4.n3.2020.207

Fong, W., Pitre, R., y Chiquillo -Rodelo, J. (2018). Significant Learning and its Association with Teaching Quality and Previous Knowledge in Engineering Students. Contemporary Engineering Sciences,11(49), 2413 2421.doi: https://doi.org/10.12988/ces.2018.85231

García-Ruíz, R., Tirado, R., y Hernando, A. (2018). Redes sociales y estudiantes: motivos de uso y gratificaciones. Evidencias para el aprendizaje. Aula Abierta,47(3),291298.Recuperado de https://dialnet.unirioja.es/servlet/articulo?codigo=6723280 
Kareiva, P., y Carranza,V. (2018). Existential Risk due to Ecosystem Collapse: Nature Strikes Back.Futures, 102,39-50.doi: https://doi.org/10.1016/j.futures.2018.01.001

Ministerio de Educación (2017). Guía de Educación Ambiental para el Desarrollo Sostenible. EsVi Espacio de Vida. (1ed.). Perú, Lima: Editorial Ministerio de Educación.

Ministerio de Educación (2017). Currículo Nacional de la Educación Básica. Lima, Perú: Editorial Ministerio de Educación.

Ministerio de Educación (2020). Guía de orientaciones para la aplicación del Enfoque Ambiental. (1ed.). Perú, Lima: Editorial Ministerio de Educación

Quillos, S., Escalante, N., Sánchez, D., Quevedo, L., y De La Cruz,R. (2018). Residuos sólidos domiciliarios: caracterización y estimación energética para la ciudad de Chimbote. Revista de la Sociedad Química del Perú,84 (3).Recuperado de http://www.scielo.org.pe/scielo.php?script=sci_arttext\&pid=S1810634X2018000300006

Rojas,J. (2017,24 de noviembre). Chimbote y la institucionalización del aburrimiento. ArchDaily. Recuperado de https://www.archdaily.pe/pe/883882/chimbote-y-lainstitucionalizacion-del-aburrimiento

Sevriche-Sierra, C., Gómez-Bustamante, E., y Jaimes-Morales, J. (2016).La educación ambiental como base cultural $\mathrm{y}$ estrategia para el desarrollo sostenible. Telos, 18

(2),266-281.Recuperado de https://www.redalyc.org/pdf/993/99345727007.pdf

Torres, L., Carbo, N., y López, J. (2017). Huella de carbono y los conocimientos, actitudes y prácticas de los estudiantes y personal del nivel secundario sobre emisiones de gases de efecto invernadero. Revista de Investigación Apuntes Universitario,7(2), 54 - 63. doi: http://dx.doi.org/10.17162/au.v7i2.171

Velásquez, G., Bedoya, M., y Cadavid, E. (2020). Estrategias didácticas y competencias ambientales desde la teoría cognitivo social: Un estudio de mapeo sistemático. Revista Boletín Redipe: Red Iberoamericana de Pedagogía,9(12),101110.Recuperado de https://revista.redipe.org/index.php/1/article/view/1138/1034 
Villanueva, H., Medina O., y Sánchez, A. (2020). Educación, cultura y comunicación ambientales. Revista Iberoamericana Ambiente \& Sustentabilidad,3 (1), 0614.doi: https://doi.org/10.46380/rias.v3i1.4

Viñan, J., Navarrete, F., Puente, M., Pino, S., y Caicedo, F. (2018). Metodología de la investigación científica como instrumento en la producción y realización de una investigación.

Atlante.

Recuperado

de https://www.eumed.net/rev/atlante/2018/05/investigacion-cientifica.html

World Health Organization (21 March 2019). Ten threats to global health in 2019.Representative Office for Viet Nam. Recovered from https://www.who.int/vietnam/news/feature-stories/detail/ten-threats-to-globalhealth-in-2019

Yangalí, J., Vásquez, M., Huaita, D., y Baldeón, M. (2020). Comportamiento ecológico y cultura ambiental, fomentada mediante la educación virtual en estudiantes de Lima-Perú. Revista de Ciencias Sociales,27 (1),385-398.Recuperado de https://produccioncientificaluz.org/index.php/rcs/article/view/35321/37413 